\title{
Image Compression using Digital Curvelet Transform and HWT as MCA
}

\author{
Navjot Kaur \\ Rayat and Bahra Institute of \\ Engineering and Biotechnology, \\ Kharar, Mohali, India
}

\author{
Deepa Verma \\ Rayat and Bahra Institute of \\ Engineering and Biotechnology, \\ Kharar, Mohali, India
}

\begin{abstract}
Image compression has been always a very active field of research. A highly efficient numerical scheme is proposed to solve the combined optimization problem posed by the model for separating images into texture and piecewise smooth parts. In the proposed multi-layered image coding schemes, the MCA used in image decomposition is performed using haar wavelet transform that decomposes the image into four frequency sub-band. The results show that the proposed algorithm that is the combination of wavelet based decomposition as extraction of texture and edge parts using the haar wavelet transform and further compressing of texture and edge part using dct and the Curvelet transform respectively, give the enhanced PSNR and other statistical parameters. The results are evaluated in different bits per pixels (bpp) color format and are in a proportionate order. i.e. as the bpp increases, the PSNR improves. Other image compression performance parameters like Standard Deviation, Entropy, Compression Ratio and Class Variance are evaluated to analyse the compression performance.
\end{abstract}

\section{Keywords}

Keywords are your own designated keywords which can be used for easy location of the manuscript using any search engines.

\section{INTRODUCTION}

Image compression is a very active field of research. The aim is to find a sparse representation for an image, so that one needs to store few coefficients to retrieve the image. The basic idea presented in the model is the use of two appraches, one for the representation of textures and the other for the natural scene parts. The proposed algorithm that is the combination of wavelet based decomposition as extraction of texture and edge parts using the haar wavelet transform and further compressing of texture using dct and edge part using the curvelet transform give the enhanced PSNR and other statistical parameters. Both approaches are chosen such that they lead to sparse representations over one type of imagecontent (either texture or piecewise smooth). The work in describes a novel multi-layered image compression algorithm based on image decomposition using the proposed MCA algorithm using the haar wavelet transform has been proved to be a very effective tool while decomposing the input image into its texture and edge parts.

\section{RELATED WORKS}

As per Changsheng Lang et.al [1], A new multi-layered representation technique for image compression is proposed, which combine curvelet transform and local DCT in order to benefit from the advantages of each. We exploit morphological component analysis (MCA) method to separate the image into two layers: piecewise smooth layer and texture structure layer, respectively associated to curvelet transform and local DCT. Each layer is encoded independently with a different transform at a different bit rate. Experiment results show that the proposed multi-layered image coding technique outperforms single curvelet transform with SPIHT in both PSNR and visual quality, with the improvement up to $0.71 \mathrm{~dB}$ on images with rich texture and edges.

As per Jing Jin Renshu Gu Jie Yuan [2] It has been studied that the Image block effect is due to the quantification process using Discrete Cosine Transform (DCT) to compression coding, which dumps some frequency, and leads to noticeable discontinuous leaps. A deblocking algorithm based on curvelet transform is proposed in this paper. This algorithm processes the curvelet coefficients separately which obtained by curvelet transform of the degraded images to recovery the images. The coefficients corresponding to block effect of the original image can be found for every layer and different layers using different methods. Then new coefficient matrixes are obtained, using which images are reconstructed.

As per Nadia Baaziz et.al [3], There have been many applications in the multiresolution representations for image processing and data compression. Several approaches have been developed on this domain using different ways, the most widely used are sub-band decompositions with filter banks and pyramid transforms. The goal of this paper is to implement a set of pyramid transforms in an hierarchical approach for real television sequence which consists on a 3-D data information set. The investigation is based on several visual and objective comparative criteria including the relevance to the motion information

As per Jean-Luc Starck et.al [4], digital transforms is applied to the denoising of some standard images embedded in white noise. In the tests reported here, simple thresholding of the curvelet coefficients is very competitive with "state of the art" techniques based on wavelets, including thresholding of decimated or undecimated wavelet transforms and also including tree-based Bayesian posterior mean methods. Existing theory for curvelet and ridgelet transforms suggests that these new approaches can outperform wavelet methods in certain image reconstruction problems.

As per Awais Mansoorand et.al[5], In this paper, a novel approach is described to digital image compression using a new mathematical transform: the curvelet transform. The transform has shown promising results over wavelet transform for 2-D signals. Wavelets, though well suited to point singularities have limitations with orientation selectivity, and therefore, do not represent two-dimensional singularities (e.g. smooth curves) effectively. This paper employs the Curvelet transform for image compression, exhibiting good approximation properties for smooth 2D functions. Curvelet improves wavelet by incorporating a directional component. In this paper, we divided 2-D spectrum into fine slices using iterated tree structured filter bank. Different amount of quantized curvelet coefficients were then selected for lossy compression and entropy encoding. 
A comparison with wavelet based compression was made for standard images like Lena, Barbara, etc. Curvelet transform has resulted in high quality image compression for natural images. Our implementation offers exact reconstruction, prone to perturbations, ease of implementation and low computational complexity. The algorithm works fairly well for grayscale and colored images.

As per M. Manikandan' et.al.[6],. In this paper an algorithm for lossy image compression based on the second generation digital curvelet transform is proposed. The results are compared with the results obtained from wavelet based image compression methods. Compression ratio and PSNR are selected as the performance metrics, and it is shown that curvelet transform require fewer coefficients than wavelet transform to represent an image faithfully.

As per Muhammad Azhar Iqbal et.al[7], In this paper, the implementation of a new compression methodology, which uses curvelet coefficients with SPIHT (Set Partitioning in Hierarchical Trees) encoding scheme is described. The first phase deals with the transformation of the stimulus image into the curvelet coefficients. During the second phase, a threshold-based selection mechanism has been developed to get prominent coefficients out of different scales. Final phase deals with the application of lossy SPIHT encoding technique on selected significant coefficients. SPIHT exploits the multiscale nature of curvelet transform and removes the statistical and subjective redundancies. The empirical results on standard test images provide higher PSNR than some of the previous approaches, which strengthen the idea of using curvelet transform instead of wavelet transform in order to get lesser bits to represent more prominent features.

\section{CURVELET TRANSFORM}

Curvelet transform is the directional transform and overcomes the limitation of the wavelet transform. The curvelet transform, like the wavelet transform, is a multiscale transform with frame elements indexed by scale and location parameters and the curvelet pyramid contains elements with a very high degree of directional specificity. The orientation selectivity behaviour and anisotropic nature of the curvelet transform helps to represent suitably the objects with curves and handles other two-dimensional singularities better than wavelets, which makes it a more proficient transformation for image compression application.

The first generation curvelet transform originally developed in the continuous domain is through multiscale filtering, followed by a block Ridgelet transform on each band pass image. Due to its computational complexity and high redundancy this technique is seldom used for image compression but find application in image denoising, image fusion etc. In order to reduce the computational complexity of the first generation curvelet transform, second generation curvelet transform was introduced. It was defined directly via frequency partitioning without using the ridgelet transform. The orientation selectivity behaviour and anisotropic nature of the curvelet transform helps to represent suitably the objects with curves and handles other two-dimensional singularities better than wavelets, which makes it a more proficient transformation for image compression application.

Later on in 2005, Candès and others proposed the Fast Discrete Curvelet Transform (FDCT). Curvelet transform, which is developed from ridgelet transform, is a multiscale geometric analytic method considered as very useful and novel. It uses the local straight lines of many different scales to express the curve approximately, and can approximate the singular curve of the image well. Curvelet transform synthesizes the merits of ridgelet transform, which is good at expressing straight line characteristics, and wavelet transform, which is suitable for point characteristics. It is applied to image intensification, image fusion, and image denoising and all of these applications demonstrated good effects.

The continuous curvelet transform can be defined by a pair of windows $\mathrm{W}(\mathrm{r})$ (a radial window) and $\mathrm{V}(\mathrm{t})$ (an angular window), with variables $\mathrm{W}$ as a frequency-domain variable, and $\mathrm{r}$ and $\theta$ as polar coordinates in the frequency-domain and are given by:

$$
\begin{aligned}
& \sum_{j=-\infty}^{\infty} \quad \mathrm{w}^{2}\left(2^{\mathrm{j}} \mathrm{r}\right)=1, \quad \mathrm{r} €\{3 / 4,3 / 2\} \\
& \sum_{j=-\infty}^{\infty} \mathrm{V}^{2}(\mathrm{t}-1)=1, \mathrm{t} €\{-1 / 2,1 / 2\}
\end{aligned}
$$

Curvelets are well-localized in time- and frequency domain. Because of their shape, they possess a high directional sensitivity. Curvelets are constructed by tiling of the frequency plane, they are complex functions

Please use a 9-point Times Roman font, or other Roman font with serifs, as close as possible in appearance to Times Roman in which these guidelines have been set. The goal is to have a 9-point text, as you see here. Please use sans-serif or non-proportional fonts only for special purposes, such as distinguishing source code text. If Times Roman is not available, try the font named Computer Modern Roman. On a Macintosh, use the font named Times. Right margins should be justified, not ragged.

\subsection{Algorithm}

The proposed algorithm is implemented using matlab tool. Followings are the steps:

- The input image is acquired either by using digital camera or browsed from the disk.

- The input image is then converted to gray scale image using the rgb2gray command in matlab and resized to $256 \times 256$.

- $\quad$ The resized image is converted to $0.1,0.3,0.5,0.7,0.9$, 1.1 and 8 bits per pixel color format using the color decomposition method.

- The haar wavelet decomposition is applied on color decomposed image in order to get LL, LH, HL and $\mathrm{HH}$ sub-band images. The LL sub-bad image is basically the approximate Image i.e. containing the maximum information in average form. We use it as a textural part of the input image. This is in actual is the MCA methodology wavelet domain to extract the texture part.

- The HH band image contains the edge information part that contains the edges. Therefore, now we have texture as well as edge separated images.

- Now, a curvelet transform is applied on HH sub band image to compress the edge part. And LL sub band image is compressed using the dct based image compression.

- Finally the compressed images from det and curvelet are combined together as both are wavelet sub bands. 
- LL and HH sub bands images are reconstructed using the inverse wavelet transform to get the final compressed image.

- PSNR, entropy, standard deviation, class variance and compression ratio are computed using the following formulas:

\subsubsection{PSNR:}

$P S N R=10 \log _{10}$

$255^{2}$

$$
\overline{\frac{1}{N x N} \sum_{i=0}^{N-1} \sum_{j=0}^{N-1}\left(\mathrm{f}(\mathrm{l}, \mathrm{j})-\mathrm{f}^{\prime}(\mathrm{l}, \mathrm{j})\right)^{2}}
$$

where $\mathrm{N} \times \mathrm{N}$ is the size of the original image and $f(i, j)$ and ${ }^{\wedge} \mathrm{f}(\mathrm{i}, \mathrm{j})$ are the gray-level pixel values of the original and reconstructed images, respectively.

Standard Deviation (SD): The standard variation of an image is given by:

$$
\sigma^{2}=\frac{1}{M x N} \sum_{i=1}^{M} \sum_{j=1}^{N}\left(\mathrm{x}_{(\mathrm{l}, \mathrm{j})}-\mu\right)^{2}
$$

Entropy E: The expression of the information entropy of an image is given by:

$$
\mathrm{H}=-\sum_{i=0}^{l-1} \mathrm{P}_{\mathrm{i}} \log \mathrm{P}_{1}
$$

Where $\mathrm{L}$ denotes the number of gray level, pi equals the ratio between the number of pixels whose gray value equals i ( 0 to $\mathrm{L}-1$ ) and the total pixel number contained in an image.

Class Variance: Variance is computed by using the matlab command $\mathrm{V}=\operatorname{var}(\mathrm{X})$ returns the variance of image vector.

\section{Compression Ratio}

Compression ratio (CR) is defined as:

$$
C R=\frac{O I S}{C I S}
$$

Where OIS $\rightarrow$ Original Image Size, CIS $\rightarrow$ Compressed Image Size.

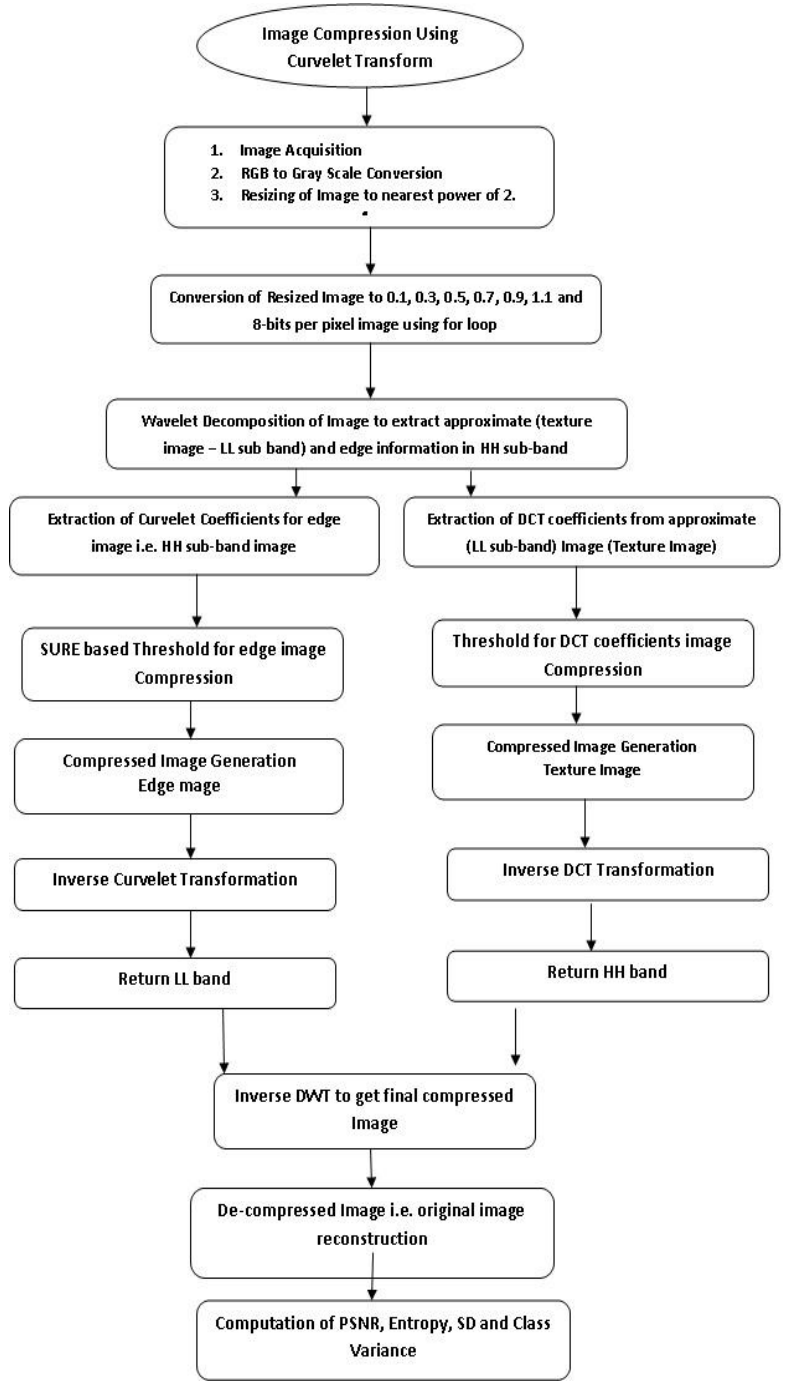

\section{RESULTS}

The proposed algorithm has been tested over a no. of images including the standard images. The proposed algorithm has shown remarkable improvements in the lossless compression of images without compromising on the quality of decompressed image. The results for the standard images are compiled in the table below.

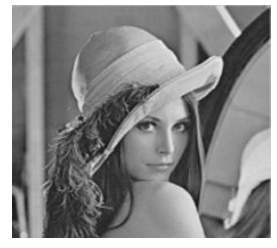

Fig 1

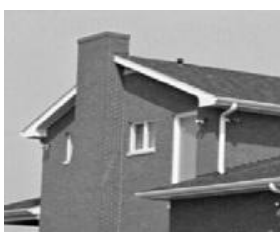

Fig 3

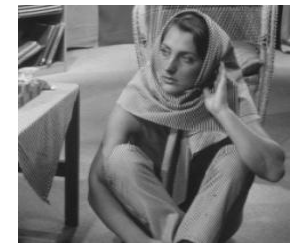

Fig 2

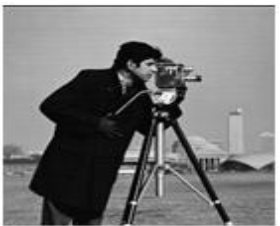

Fig 4 
Table -1 (Leena)

\begin{tabular}{|c|c|c|c|c|c|c|}
\hline BPP & MSE & PSNR & SD & CV & Entropy & CR \\
\hline 0.1 & 653.065 & 55.304 & 25.555 & 0.01 & 1.228 & 1.256 \\
\hline 0.3 & 640.004 & 55.547 & 24.616 & 0.009 & 3.188 & 1.248 \\
\hline 0.5 & 627.204 & 55.789 & 24.191 & 0.009 & 3.876 & 1.297 \\
\hline 0.7 & 614.66 & 56.031 & 24.092 & 0.009 & 4.486 & 1.325 \\
\hline 0.9 & 602.366 & 56.274 & 23.677 & 0.009 & 4.892 & 1.328 \\
\hline 1.1 & 590.319 & 56.516 & 20.452 & 0.006 & 6.175 & 1.328 \\
\hline 8 & 578.513 & 56.759 & 23.978 & 0.009 & 7.478 & 1.333 \\
\hline
\end{tabular}

Table -2 (Barbara)

\begin{tabular}{|c|c|c|c|c|c|c|}
\hline BPP & MSE & PSNR & SD & CV & Entropy & CR \\
\hline 0.1 & 594.148 & 56.439 & 24.375 & 0.009 & 1.14 & 1.215 \\
\hline 0.3 & 582.265 & 56.681 & 23.445 & 0.008 & 3.046 & 1.216 \\
\hline 0.5 & 570.62 & 56.924 & 23.045 & 0.008 & 3.793 & 1.26 \\
\hline 0.7 & 559.207 & 57.166 & 23.031 & 0.008 & 4.416 & 1.287 \\
\hline 0.9 & 548.023 & 57.408 & 22.925 & 0.008 & 4.891 & 1.3 \\
\hline 1.1 & 537.063 & 57.651 & 21.663 & 0.007 & 6.521 & 1.306 \\
\hline 8 & 526.322 & 57.893 & 22.983 & 0.008 & 7.394 & 1.306 \\
\hline
\end{tabular}

Table -3 (House)

\begin{tabular}{|c|c|c|c|c|c|c|}
\hline BPP & MSE & PSNR & SD & CV & Entropy & CR \\
\hline 0.1 & 539.923 & 57.587 & 23.236 & 0.008 & 1.463 & 1.28 \\
\hline 0.3 & 529.124 & 57.83 & 23.726 & 0.009 & 2.849 & 1.283 \\
\hline 0.5 & 518.542 & 58.072 & 22.72 & 0.008 & 3.17 & 1.325 \\
\hline 0.7 & 508.171 & 58.314 & 23.005 & 0.008 & 3.884 & 1.327 \\
\hline 0.9 & 498.008 & 58.557 & 23.089 & 0.008 & 4.005 & 1.326 \\
\hline 1.1 & 488.047 & 58.799 & 17.908 & 0.005 & 5.09 & 1.326 \\
\hline 8 & 478.286 & 59.042 & 23.365 & 0.008 & 6.601 & 1.327 \\
\hline
\end{tabular}

Table -4 (Cameraman)

\begin{tabular}{|c|c|c|c|c|c|c|}
\hline BPP & MSE & PSNR & SD & CV & Entropy & CR \\
\hline 0.1 & 567.785 & 56.983 & 23.828 & 0.009 & 1.147 & 1.255 \\
\hline 0.3 & 556.429 & 57.226 & 27.419 & 0.011 & 2.455 & 1.266 \\
\hline 0.5 & 545.3 & 57.468 & 27.537 & 0.012 & 3.075 & 1.294 \\
\hline 0.7 & 534.394 & 57.711 & 27.475 & 0.012 & 3.809 & 1.306 \\
\hline 0.9 & 523.706 & 57.953 & 27.212 & 0.011 & 3.98 & 1.311 \\
\hline 1.1 & 513.232 & 58.196 & 24.549 & 0.009 & 4.092 & 1.308 \\
\hline 8 & 502.968 & 58.438 & 27.379 & 0.011 & 6.629 & 1.312 \\
\hline
\end{tabular}

\section{CONCLUSION}

The results show that the proposed algorithm that is the combination of wavelet based decomposition as extraction of texture and edge parts using the haar wavelet transform and further compressing of texture using dct and edge part using the curvelet transform give the enhanced PSNR and other statistical parameters. This proves the effectiveness of the proposed algorithm over the base work used as references. The proposed MCA algorithm using the haar wavelet transform has been proved to be a very effective tool while decomposing the input image into its texture and edge parts. The results are evaluated in different bits per pixels color format and are in a proportionate order. i.e. as the bpp increases, the PSNR improves.

\section{REFERENCES}

[1] Changsheng Lang, Hong LI, Guangzheng LI, Xiujuan ZHAO "Combined Sparse Representation Based on Curvelet Transform and Local DCT for Multi-layered Image Compression" 978-1-61284-486-2/11/\$26.00 (C)2011 IEEE.

[2] Jing Jin Renshu Gu Jie Yuan “A Novel Image Deblocking Method Based on Curvelet Transform" 2011 Seventh International Conference on Natural Computation 978-1-4244-9953-3/11 @2011 IEEE.

[3] Nadia Baaziz and Claude Labit "Laplacian Pyramid Versus Wavelet Decomposition for Image Sequence Coding" 1990 IEEE

[4] Jean-Luc Starck, Emmanuel J. Candès, and David L. Donoho. "The Curvelet Transform for Image Denoising" IEEE TRANSACTIONS ON IMAGE PROCESSING, VOL. 11, NO. 6, JUNE 2002.

[5] Awais Mansoorand AtifBin Mansoor. "ON IMAGE COMPRESSION USING DIGITAL CURVELET TRANSFORM" Center for Advanced Studies in Engineering (CASE), University ofEngineering and Technology, Taxila-Pakistan.

[6] M. Manikandan', A. Saravanan' and K. Bhoopathy Bagan2 "Curvelet Transform Based Embedded Lossy Image Compression" IEEE - ICSCN2007, MITCampus, Anna University, Chennai, India. Feb. 22-24, 2007. pp.274-276.

[7] Muhammad Azhar Iqbal, Dr Muhammad Younus Javed, Usman Qayyum "Curvelet-based Image Compression with SPIHT" 2007 International Conference on Convergence Information Technology.

[8] Yuancheng Li, Qiu Yang, Runhai Jiao “A Novel Image Compression Algorithm Using the Second Generation of Curvelet Transform and SVM" 978-0-7695-3571-5/09 2009 IEEE.

[9] Rafeeq Al- Hashemi, Israa Wahbi Kamal; "A New lossless Image Compression Technique based on Bose, Chandhuri and Hocquengham $(\mathrm{BCH})$ Codes", International Journal of Software Engineering and its Applications, Vol.5 No.3, july,2011.

[10] Lifeng Xi, Liangbin Zhang; "A Study of Fractal Image Compression based on an Improved Genetic Algorithm", International Journal of Nonlinear Science, Vol.3(2007) No.2,pp. 116-124

[11] Y. Chakrapani and K. Soundara Rajan; " Genetic Algorithm Applied to Fractal Image Compression", 
ARPN Journal of Engineering and Applied Sciences; Vol.4, No.1, February 2009.

[12] Samir Kumar Bandyopadhyay, Tuhin Utsab Paul, Avishek Raychoudhury; "Image Compression using Approximate Matching and Run Length", International Journal of Advanced Computer Science and Applications(IJACSA), Vol.2, No.6, 2011.

[13] K. John Singh and R. Manimegalai, "A Survey on Joint Compression and Encryption Techniques for Video Data”, Journal of Computer Science 8(5): 731-736, 2012.

[14] Jagadish H. Pujar, Lohit M. Kadlaskar; “ A new Lossless Method of Image Compression and Decompression using
Huffman Coding Techniques"; Journal of Theoretical and Applied Information Technology.

[15] Dr. B Eswara Reddy and K Venkata Narayana; “ A Lossless Image Compression using Traditional and Lifting based Wavelets" Signal and Image Processing: An International Journal(SIPIJ) Vol.3, No.2, April 2012.

\section{AUTHOR PROFILE}

Navjot Kaur received the B.Tech. in ECE from GGSCET, Talwandi Sabo (Punjab) in 2011. Her field of interest. is in the developing the systems based on image processing applications. Presently she is pursuing het masters in ECE from RBIEBT, Mohali. 